\title{
Febre Escaro-Nodular: Revisão Retrospetiva de Casos Hospitalizados e Fatores Preditores de Doença Severa
}

\author{
Mediterranean Spotted Fever: Retrospective Review of Hospitalized Cases \\ and Predictive Factors of Severe Disease
}

\author{
Mariana MEIRELES $\rrbracket^{1}$, Rui MAGALHÃES ${ }^{2}$, Arlindo GUIMAS ${ }^{3}$ \\ Acta Med Port 2015 Sep-Oct;28(5):624-631
}

\section{RESUMO}

Introdução: Febre escaro-nodular, uma zoonose antiga a levantar questões actuais. Este estudo pretende reavaliar a imagem clínica da doença e identificar factores prognósticos de doença severa.

Material e Métodos: Foram avaliados os casos admitidos num hospital central no período de 12 anos. Os fatores de risco foram determinados por análise uni e multivariada, comparando os indivíduos internados em enfermaria geral versus admitidos em unidades de cuidados intermédios/intensivos ou em caso de morte.

Resultados: Foram revistos 71 casos de febre escaro-nodular. A média de idades foi $63,3 \pm 16,7$ anos e $52,1 \%$ eram homens. A escara de inoculação foi identificada em $62,0 \%$ dos casos. Febre e exantema foram os achados clínicos mais frequentes. Quarenta e cinco por cento dos doentes desenvolveram complicações e $22,5 \%$ foram internados em unidades de cuidados intermédios e/ou intensivos. Apirexia, dispneia, insuficiência renal e níveis de lactato desidrogenase elevados à admissão foram preditores de desenvolvimento de doença severa $(p<0,034)$. O alcoolismo associou-se a um tempo de internamento prolongado $(p=0,020)$. Ausência de febre $(p=0,019)$ e níveis elevados de creatinina $(p=0,028)$ constituíram fatores independentes de mau prognóstico. A taxa de mortalidade foi $2,8 \%$.

Discussão: Apesar da evolução da febre escaro-nodular ser, geralmente, assumida como benigna, casos graves têm surgido. Quase metade dos doentes hospitalizados desenvolveu complicações. Apirexia e lesão renal predispõem a pior prognóstico.

Conclusão: A identificação precoce da infeção e a vigilância contínua dos doentes são determinantes, particularmente naqueles com maior risco de desenvolverem doença severa ou fatal.

Palavras-chave: Factores de Risco; Febre escaro-nodular; Infecções por Rickettsia; Prognóstico; Rickettsia conorii.

\section{ABSTRACT}

Introduction: Mediterranean spotted fever, an ancient zoonotic disease raising current issues. This study aims to reevaluate the disease clinical picture and to identify prognostic factors related to severe disease.

Material and Methods: We evaluate cases admitted to a central hospital during 12-year period. The risk factors were determined by uni and multivariate analysis, comparing patients admitted in general infirmary versus intermediate/intensive care units or in case of death.

Results: We reviewed 71 cases of Mediterranean spotted fever. The mean age was $63.3 \pm 16.7$ years and $52.1 \%$ were male. The eschar was observed in $62.0 \%$. Fever and rash were the most common clinical findings. Forty five percent of patients developed complications and $22.5 \%$ were admitted to intermediate and/or intensive care units. Apyrexia, dyspnea, renal failure and elevated lactic dehydrogenase levels at admission were predictors of severe disease development $(p<0.034)$. Alcoholism was related with prolonged hospitalization $(p=0.020)$. Absence of fever $(p=0.019)$ and elevated serum creatinine levels $(p=0.028)$ were identified as independent factors associated with severe outcome. The mortality rate was $2.8 \%$.

Discussion: Although Mediterranean spotted fever is usually assumed to be benign, severe cases are emerging.

Conclusion: Early identification of the infection and continuous patient surveillance are crucial, particularly in those with greater risk of developing severe or fatal disease.

Keywords: Boutonneuse Fever; Prognosis; Rickettsia conorii; Rickettsia Infections; Risk Factors.

\section{INTRODUÇÃO}

A febre escaro-nodular (FEN) é uma zoonose endémica nos países da orla mediterrânica. Segundo dados da Direção Geral de Saúde, entre 2000 e 2010 foram notificados 3,72 casos $/ 10^{5}$ habitantes/ano. ${ }^{1,2}$

Em Portugal, as principais estirpes responsáveis pela doença são a Rickettsia conorii Malish e a $R$. conorii Israeli spotted fever (ISF), ${ }^{3}$ a última descrita pela primeira vez, no nosso país, em 1997. ${ }^{4}$ As manifestações clínicas e laboratoriais são resultado de uma vasculite sistémica de pequenos e médios vasos ${ }^{5}$ consequente ao angiotropismo próprio do agente. Classicamente a doença caracteriza-se pela tríade febre alta, escara de inoculação e exantema maculopapular. ${ }^{6,7}$

$\mathrm{Na}$ região mediterrânica, o interesse por esta entidade clínica foi reforçado nos últimos anos. No final da década de 80 e ao longo dos anos 90 , o número de casos de FEN declarados em Portugal aumentou drasticamente, tendo este número sido superior a 1000 por ano em 1987 , no período 1989 - 1992 e em 1996. ${ }^{1,2,8}$ O recrudescimento desta doença é explicado, por alguns autores, pelo aumento das

1. Serviço de Medicina Interna. Centro Hospitalar do Porto. Porto. Portugal.

2. UNIFAI. Departamento de Estudos de Populações. Instituto de Ciências Biomédicas de Abel Salazar. Universidade do Porto. Porto. Portugal.

3. Unidade de Cuidados Intermédios Médico-Cirúrgicos. Centro Hospitalar do Porto. Porto. Portugal.

$\square$ Autor correspondente: Mariana Meireles. mra.meireles@gmail.com

Recebido: 16 de Novembro de 2014 - Aceite: 12 de Agosto de 2015 | Copyright @ Ordem dos Médicos 2015 
temperaturas médias e diminuição da precipitação, fatores que favorecem a atividade do vetor, o Rhipicephalus sanguineus, permitindo ao artrópode completar mais do que dois ciclos de vida por ano. ${ }^{9}$ Fatores como a movimentação da população rural para as regiões urbanas e suburbanas podem ter deslocado as clássicas fontes de infeção para estes territórios, ${ }^{10}$ tornando a patologia mais visível. Por outro lado, a maior deteção de casos relacionada com o advento dos novos métodos de diagnóstico pode estar na sua génese.

Em 1982, Raoult et al descreve os primeiros de casos malignos de FEN. ${ }^{10}$ Formas severas, incluindo manifestações neurológicas graves e disfunção multiorgânica, estão descritas em cinco a $6 \%$ dos casos, com uma taxa de mortalidade associada de 2,5\%. ${ }^{11}$ Entre 1994 e 2004, o número de hospitalizações em Portugal passou de 176 casos para 446 casos por ano, ${ }^{12}$ respetivamente, com aumento significativo do número de casos fatais (1,5\% em 1995 vs 2,6\% em 1999). ${ }^{3}$ Este facto foi particularmente notório no Hospital Distrital de Beja e no Hospital Garcia de Orta onde, em 1997 , a taxa de mortalidade atingiu $32 \%$ e $18 \%$, respectivamente. ${ }^{4}$

Nos últimos 20 anos têm surgido, por toda a Europa, novas espécies de Rickettsia, algumas das quais já descritas em Portugal. ${ }^{10,13,14}$ Questiona-se se estas estirpes emergentes serão mais virulentas. ${ }^{12}$ Por outro lado, a descrição recente e paralela de casos atípicos, ${ }^{3,11}$ onde a escara e o exantema são características ausentes, ${ }^{15}$ tornam o seu reconhecimento mais difícil para os clínicos, atrasando as medidas terapêuticas e comprometendo o seu desfecho.

São reconhecidos diversos fatores de mau prognóstico, entre os quais a idade avançada; a presença de comorbilidades como a diabetes mellitus, o alcoolismo, a imunossupressão e o défice de glicose-6-fosfato-desidrogenase; e o atraso na instituição da terapêutica adequada. ${ }^{12,16,17}$ Diversos estudos demonstraram uma associação positiva entre a morte e a apresentação clínica com sintomas gastrointestinais, prostração, exantema petéquial, azotemia e alterações das enzimas e função hepáticas. ${ }^{12,16,17}$

O aumento do número de casos de FEN documentados nas últimas décadas, bem como as crescentes dúvidas em relação à sua benignidade, tornam relevante a reavaliação da patologia.

Os objetivos deste estudo passam pela caracterização epidemiológica da doença e do padrão actual de apresentação num hospital urbano e central e a discriminação de fatores de mau prognóstico.

\section{MATERIAL E MÉTODOS}

Trata-se de um estudo retrospetivo de base hospitalar. Foram sinalizados os casos de infeção por Rickettsia internados no Hospital de Santo António - Centro Hospitalar do Porto (HSA-CHP) entre 1 de janeiro de 1995 e 31 de dezembro de 2011 com base na classificação ICD9 (ICD9-CM 0,82 a 0,839 ), tendo sido selecionados os que cumpriam os seguintes critérios: idade igual ou superior a 18 anos; diag- nóstico clínico (febre, escara e exantema), e/ou serológico (técnicas de IF ou ELISA), e/ou histológico.

Foram recolhidos dados epidemiológicos, clínicos, laboratoriais, terapêuticos, particularidades do internamento e destino, com recurso ao processo clínico electrónico e em papel.

Foram consideradas como comorbilidades o atingimento dos sistemas cardiovascular (considerando insuficiência cardíaca, disritmia e doença vascular crónicas); respiratório (doença pulmonar ou insuficiência respiratória crónicas); sistema nervoso central (antecedentes de acidente vascular cerebral ou doenças degenerativas); imunodeficiências primárias e adquiridas. Alcoolismo foi assumido quando ingestão superior a $100 \mathrm{~g} / \mathrm{dia}$ ou se referência no processo. A data de início dos sintomas foi definida como o primeiro dia de aparecimento de um dos seguintes sintomas/sinais: febre, arrepios, exantema, mialgias, cefaleias, náuseas, vómitos e/ou diarreia. Os dados laboratoriais avaliados foram os correspondentes ao dia da admissão no serviço de urgência. De entre as complicações, febre prolongada foi definida como a persistência após três dias de antibioterapia; complicações do SNC incluíram diagnóstico de convulsões, meningite, encefalite e mielite; complicações cardiovasculares em caso de hipotensão ou choque; e respiratórias se objectivação de insuficiência respiratória, definida como $\mathrm{PaO}_{2}<60 \mathrm{mmHg}$. O internamento foi considerado prolongado quando superior a 10 dias.

Os doentes identificados foram divididos consoante a admissão em enfermaria geral (grupo 1) ou em unidades de cuidados intermédios/intensivos e/ou morte (grupo 2). A severidade da doença e fatores associados a maior gravidade foram avaliados através da comparação dos dois grupos.

\section{Métodos Estatísticos}

A análise estatística foi realizada com recurso ao programa SPSS (versão 17.0). As variáveis categóricas foram avaliadas com o teste do Qui-quadrado de Pearson ou teste exato de Fisher, quando aplicável. As variáveis contínuas foram categorizadas, de acordo com valores de corte comummente aceites, excepto quando aplicados os modelos de regressão logística, e avaliadas pelo teste Mann-Whitney.

Para identificar os factores de risco foram aplicados modelos de regressão logística. O modelo multivariado foi construído pelo método stepwise (Forward $L R$ ), tendo sido consideradas as variáveis com um valor de $p<0,1$ no univariado e com valores desconhecidos $<10 \%$ da amostra, no sentido de identificar fatores independentes associados a internamento em unidades de cuidados intermédios/intensivos ou morte.

As variáveis foram transformadas quando não cumpriam a premissa de normalidade.

Considerou-se que existia uma associação para um valor de $p<0,05$. 


\section{Ética}

O presente estudo obteve parecer favorável do Conseção do Departamento de Ensino, Formação e Investigação/ DEFI, Direção Clínica e Comissão de Ética para a Saúde do Centro Hospitalar do Porto.

\section{RESULTADOS}

Foram confirmados 71 casos de FEN no período de 12 anos. Sessenta e oito por cento eram residentes no distrito do Porto, $25 \%$ no de Viseu e os restantes noutros distritos da região norte e centro do país de forma equitativa.

Verificou-se maior número de diagnósticos nos anos 2006 e 2010 (Fig. 1). A maioria dos casos (78,9\%) ocorreu entre julho e setembro, tendo agosto sido o mês com maior incidência. Não se registaram casos entre janeiro e março (Fig. 2).

Cinquenta e cinco indivíduos $(77,5 \%)$ foram internados numa enfermaria geral e $16(22,5 \%)$ em unidades de cuidados intermédios e/ou intensivos.

A média de idades foi $63,3 \pm 16,7$ anos (25 - 92 anos), com uma distribuição semelhante nos grupos 1 e 2 . Os doentes com idades entre os 55 e os 85 anos correspondiam a $67,6 \%$ dos casos. A proporção de homens foi $52,1 \%$.

O início dos sintomas ocorreu, em média, 7,1 $\pm 4,1$ dias antes da apresentação no serviço de urgência $(7,1 \pm 4,6$ vs $6,8 \pm 3,9$, grupos 1 e 2 , respectivamente; $p>0,9$ ). Iho de Administração, Gabinete Coordenador de Investiga-

O achado semiológico mais consistente foi o exantema, que em $67,6 \%$ atingiu as superfícies palmares e plantares. A escara de inoculação foi observada em $62,0 \%$ dos doentes. Febre, mialgias e prostração foram sintomas frequentes. O diagnóstico clínico foi obtido em $62,0 \%$ dos casos, $63,0 \%$ no grupo 1 e $58,8 \%$ no grupo 2 ( $p>0,7)$. Os restantes diagnósticos foram efectuados através de testes serológicos e, num caso, por biópsia cutânea. A alteração analítica mais frequente foi a elevação da desidrogenase láctica sérica $(\mathrm{DHL})(93,5 \%)$, seguida da trombocitopenia $(69,6 \%)$, hipoalbuminemia $(57,7 \%)$, hiponatremia $(57,1 \%)$ e prolongamento do tempo tromboplastina parcialmente ativado (APTT) (52,9\%).

A apresentação clínica e laboratorial e a sua relação com o serviço de internamento/desfecho fatal são sumariadas na Tabela 1. Não se observaram diferenças em termos de comorbilidades nos dois grupos. Os doentes do grupo 2 apresentaram-se mais frequentemente apiréticos $(p=$ $0,010)$ e dispneicos $(p=0,020)$. A restante apresentação clínica foi semelhante nos dois grupos. Analiticamente, elevações da ureia $(p=0,033)$, creatinina $(p=0,003)$ e DHL $(p=0,004)$ foram mais frequentes no grupo 2 .

A Tabela 2 apresenta os resultados da análise uni e multivariada. Nenhuma comorbilidade se associou a maior o risco de admissão em unidades de cuidados intensivos/ intermédios ou morte. Constataram-se associações entre a presença de dispneia, os níveis séricos de DHL e creatinina

12

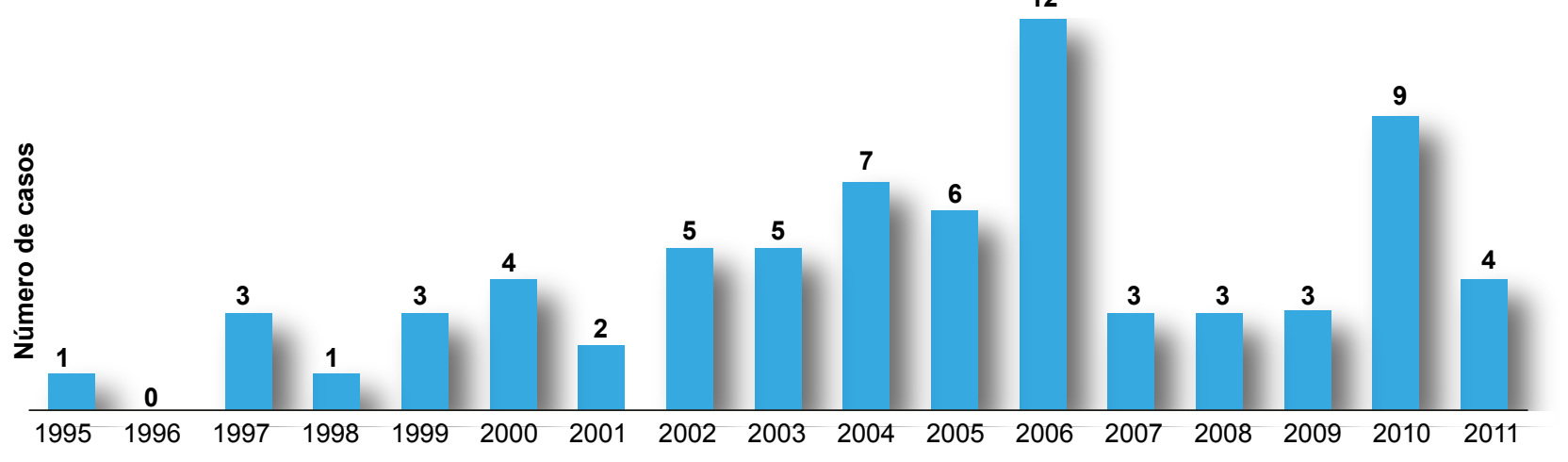

Ano

Figura 1 - Distribuição anual dos casos de FEN diagnosticados no HSA-CHP no período 1995-2011

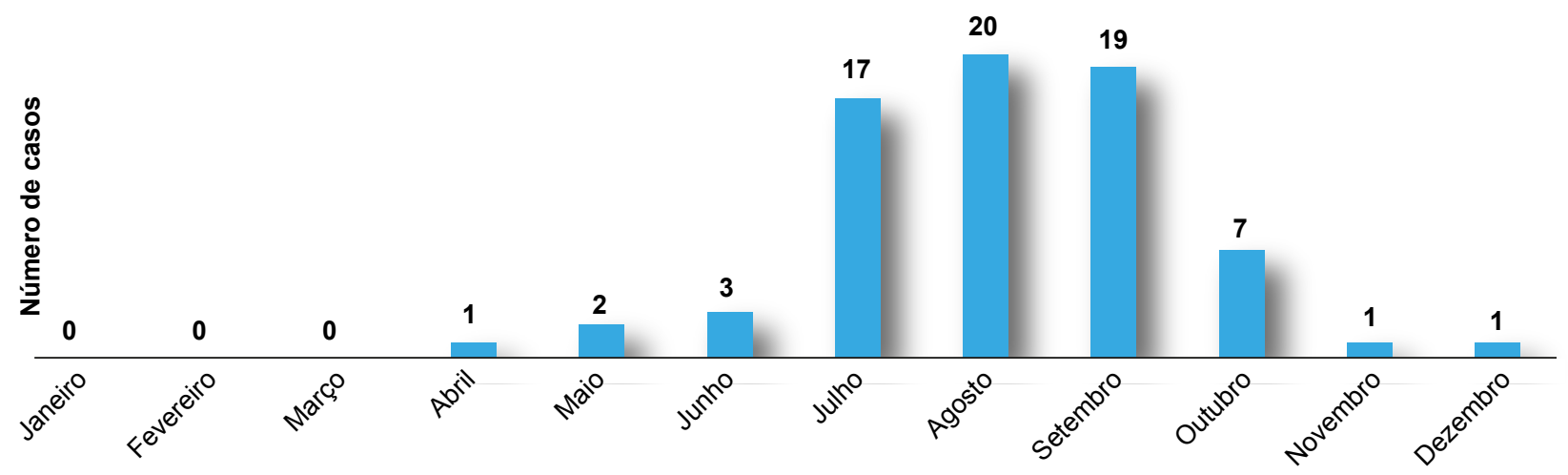

Mês

Figura 2 - Distribuição sazonal dos casos de FEN diagnosticados no HSA-CHP no período 1995-2011 
Tabela 1 - Apresentação clínica e laboratorial no serviço de urgência dos doentes internados em unidades de cuidados intermédios/ intensivos ou morte (grupo 2) versus enfermaria geral (grupo 1) - (continua)

\begin{tabular}{|c|c|c|c|c|c|c|c|}
\hline \multirow{2}{*}{ Característica } & \multicolumn{2}{|c|}{ Total } & \multicolumn{2}{|c|}{ Grupo $1(n=54)$} & \multicolumn{2}{|c|}{ Grupo $2(n=17)$} & \multirow{2}{*}{$p$} \\
\hline & $\mathrm{N}$ & $\%$ & $\mathrm{n}$ & $\%$ & $\mathrm{n}$ & $\%$ & \\
\hline \multicolumn{8}{|l|}{ Comorbilidades* } \\
\hline Obesidade & 38 & 13,2 & 3 & 5,6 & 2 & 11,8 & 1,000 \\
\hline Diabetes & 71 & 22,5 & 11 & 20,4 & 5 & 29,4 & 0,656 \\
\hline Dislipidemia & 71 & 26,8 & 15 & 27,8 & 4 & 23,5 & 1,000 \\
\hline Hipertensão arterial & 71 & 38,0 & 18 & 33,3 & 9 & 52,9 & 0,244 \\
\hline Doença cardiovascular & 71 & 28,2 & 13 & 24,1 & 7 & 41,2 & 0,290 \\
\hline Doença pulmonar & 71 & 8,5 & 3 & 5,6 & 3 & 17,6 & 0,144 \\
\hline Doença SNC & 71 & 16,9 & 11 & 20,4 & 1 & 5,9 & 0,270 \\
\hline Imunodeficiência/ Imunossupressão & 71 & 5,6 & 3 & 5,6 & 1 & 5,9 & 1,000 \\
\hline Alcoolismo & 68 & 17,6 & 9 & 17,6 & 3 & 17,6 & 1,000 \\
\hline Tabagismo & 69 & 24,6 & 16 & 30,8 & 1 & 6,9 & 0,052 \\
\hline \multicolumn{8}{|l|}{ N..$^{\circ}$ de Comorbilidades ${ }^{\dagger}$} \\
\hline 0 & 70 & 15,7 & 7 & 13,2 & 4 & 23,5 & 0,315 \\
\hline 1 & 69 & 37,7 & 21 & 40,4 & 5 & 29,4 & 0,420 \\
\hline 2 & 69 & 21,7 & 12 & 23,1 & 3 & 17,6 & 0,639 \\
\hline$\geq 3$ & 71 & 23,9 & 12 & 22,2 & 5 & 29,4 & 0,546 \\
\hline \multicolumn{8}{|l|}{ Apresentação Clínica* } \\
\hline Febre & 66 & 93,0 & 53 & 98,1 & 13 & 76,5 & 0,010 \\
\hline Escara & 71 & 62,0 & 34 & 63,0 & 10 & 58,8 & 0,984 \\
\hline Exantema maculopapular & 71 & 80,3 & 45 & 83,3 & 12 & 70,6 & 0.249 \\
\hline Exantema petequial \pm maculopapular & 71 & 15,5 & 6 & 11,5 & 5 & 31,2 & 0,069 \\
\hline Mialgias & 70 & 48,6 & 26 & 49,1 & 8 & 47,1 & 1.000 \\
\hline Artralgias & 71 & 11,3 & 7 & 13,0 & 1 & 5,9 & 0,670 \\
\hline Náuseas & 71 & 16,9 & 11 & 20,4 & 1 & 5,9 & 0,270 \\
\hline Vómitos & 71 & 22,5 & 15 & 27,8 & 1 & 5,9 & 0,094 \\
\hline Dor abdominal & 71 & 14,1 & 9 & 16,7 & 1 & 5,9 & 0,433 \\
\hline Dispneia & 71 & 16,9 & 6 & 11,1 & 6 & 35,3 & 0,020 \\
\hline Edema & 71 & 11,3 & 5 & 9,3 & 3 & 17,6 & 0,387 \\
\hline Cefaleias & 71 & 31,0 & 20 & 37,0 & 2 & 11,8 & 0,071 \\
\hline Confusão & 71 & 22,5 & 11 & 20,4 & 5 & 29,4 & 0,656 \\
\hline Prostração & 71 & 42,3 & 22 & 40,7 & 8 & 47,1 & 0,858 \\
\hline
\end{tabular}

N: Número de casos com dados disponíveis; SNC: Sistema nervoso central; AST: Aspartato aminotransferase; ALT: Alanina aminotransferase; PT: Tempo de protrombina; APTT: Tempo de tromboplastina parcialmente activado; CK: Creatinina quinase; DHL: Lactato desidrogenase sérica; PCR: Proteína C reativa; ${ }^{*}$ Doença renal crónica, neoplasia, diarreia, cianose e hepatomegalia não cumprem requisitos da análise; ${ }^{\dagger}$ Exclui comorbilidades obesidade e dislipidemia; ${ }^{\ddagger} \mathrm{nXLSN}$ : Número de vezes acima do limite superior do normal.

\section{e o grupo 2.}

A análise multivariada identificou duas variáveis como fatores independentes para internamento em unidades de cuidados intermédios/intensivos ou morte: ausência de febre $(p=0,017)$ e o aumento da ureia sérica $(p=0,021)$.

À admissão, $40,8 \%$ dos doentes tinham sido previamente medicados com terapêutica antibiótica, $27,6 \%$ dos quais com doxiciclina. Não se encontrou associação entre este facto e o local de internamento $(p>0,7)$.
Durante o internamento, $69,6 \%$ dos casos foram medicados com doxiciclina isolada e $27,5 \%$ com doxiciclina associada a outro antibiótico. Houve um caso medicado com cloranfenicol e outro com cefotaxime e rifampicina. A terapêutica antimicrobiana múltipla, embora não significativa, foi mais frequente no grupo $2(27,3 \%$ vs $35,7 \%, p>0,5)$.

As durações médias do tratamento e do internamento foram $9,8 \pm 3,1$ dias $(9,3 \pm 4,9$ vs $16,7 \pm 15,2 ; p=0,026)$ e $10,9 \pm 8,6$ dias $(9,3 \pm 2,7$ vs $11,6 \pm 4,1 ; p=0,034)$, 
respectivamente, ambas superiores no grupo 2. Quando comparados os fatores de risco para internamento prolongado apenas o alcoolismo demonstrou uma associação positiva (OR = 4,93 [IC 95\% 1,29 - 18,88]; $p=0,020$ ).

Trinta e dois doentes apresentaram pelo menos uma complicação. Febre prolongada foi observada em 22,9\%; complicações respiratórias, cardiovasculares e SNC em $17,5 \%, 15,5 \%$ e $12,7 \%$, respectivamente; icterícia em 3,3\%; celulite em $2,8 \%$ dos casos. As complicações cardiovasculares foram mais frequentes no grupo 2 (7,4\% vs 41,2\%; $p=0,003$ ) não se registando diferenças nas restantes.

Relativamente ao destino dos doentes, 5,6\% tiveram alta para outras instituições de apoio. Registaram-se dois casos de morte (2,8\%). O tempo de apresentação no serviço de urgência após início da sintomatologia foi cinco dias nos casos com desfecho fatal. Ambos apresentaram febre e exantema maculopapular à entrada mas em nenhum foi identificada escara de inoculação. Um dos casos ocorreu numa mulher de 92 anos com múltiplas comorbilidades, admitida no serviço de urgência dispneica e com insuficiência renal, com internamento durante 12 dias em enfermaria geral complicado de febre prolongada e morte. O outro caso tratava-se de um homem de 42 anos, com antecedentes de etilismo crónico, que se apresentou no serviço de urgência com disfunções respiratória, hepática e hematológica, desenvolvimento posterior de complicações cardiovasculares e febre prolongada, tendo estado internado numa unidade de cuidados intensivos durante 51 dias.

\section{DISCUSSÃO}

De acordo com a Direção Geral de Saúde, nos últimos 10 anos, tem-se observado uma diminuição do número de casos de FEN declarados em Portugal. ${ }^{1,2} \mathrm{O}$ número de casos diagnosticados no HSA-CHP no período 2002 - 2011 não acompanhou esta tendência. Em 2006, foram registados na zona norte 61 casos de FEN, 12 dos quais declarados no distrito do Porto, ${ }^{1}$ o mesmo número de casos diagnosticado neste centro. Tendo em conta o número de instituições hospitalares do distrito, bem como o facto da maioria dos casos ser tratada em ambulatório, é fácil corroborar as conclusões de alguns autores que estimam que o número de casos de FEN seja, na realidade, sete vezes superior ao declarado. ${ }^{13}$

Dois mil e dez foi um dos anos em que se diagnosticou maior número de casos neste centro. Apesar dos números nacionais não acompanharem o registo local, observou-se um número significativamente superior de casos na zona norte em relação à média da década pregressa, tendo a

Tabela 1 - Apresentação clínica e laboratorial no serviço de urgência dos doentes internados em unidades de cuidados intermédios/ intensivos ou morte (grupo 2) versus enfermaria geral (grupo 1) - (fim)

\begin{tabular}{|c|c|c|c|c|c|c|c|}
\hline \multirow{2}{*}{ Característica } & \multicolumn{2}{|c|}{ Total } & \multicolumn{2}{|c|}{ Grupo $1(n=54)$} & \multicolumn{2}{|c|}{ Grupo $2(n=17)$} & \multirow{2}{*}{$p$} \\
\hline & $\mathrm{N}$ & $\%$ & $\mathrm{n}$ & $\%$ & $\mathrm{n}$ & $\%$ & \\
\hline \multicolumn{8}{|l|}{ Dados Laboratoriais } \\
\hline Leucócitos $>11 \times 10^{3} / \mu \mathrm{L}$ & 70 & 25,7 & 13 & 24,1 & 5 & 31,2 & 0.564 \\
\hline Leucócitos $<4 \times 10^{3} / \mu \mathrm{L}$ & 70 & 8,6 & 4 & 7,4 & 2 & 12,5 & 0.523 \\
\hline Plaquetas $<150 \times 10^{3} / \mu \mathrm{L}$ & 69 & 69,6 & 35 & 66,0 & 13 & 81,2 & 0,356 \\
\hline AST $>3 \times \operatorname{LSN}^{\ddagger}(\mathrm{U} / \mathrm{L})$ & 69 & 42,0 & 20 & 37,7 & 9 & 56,2 & 0.189 \\
\hline ALT > $3 \times \operatorname{LSN}^{\ddagger}(\mathrm{U} / \mathrm{L})$ & 68 & 42,0 & 20 & 37,7 & 9 & 56,2 & 0.189 \\
\hline Bilirrubina total $>2 \mathrm{mg} / \mathrm{dL}$ & 60 & 11,7 & 5 & 10,9 & 2 & 14,3 & 0.660 \\
\hline Gama-GT > 3 X LSN L $^{(\mathrm{U} / \mathrm{L})}$ & 28 & 46,4 & 10 & 41,7 & 3 & 75,0 & 0,311 \\
\hline Albumina $<3,5 \mathrm{~g} / \mathrm{dL}$ & 26 & 57,7 & 10 & 47,6 & 5 & 100,0 & 0.053 \\
\hline PT > 13 segundos & 34 & 41,2 & 8 & 34,8 & 6 & 54,5 & 0.273 \\
\hline APTT > 35 segundos & 34 & 29,4 & 6 & 26,1 & 4 & 36,8 & 0.692 \\
\hline Creatinina $>1,2 \mathrm{mg} / \mathrm{dL}$ & 69 & 30,4 & 11 & 21,2 & 10 & 58,8 & 0,003 \\
\hline Ureia $>60 \mathrm{mg} / \mathrm{dL}$ & 70 & 37,1 & 16 & 30,2 & 10 & 58,8 & 0.033 \\
\hline Sódio < $135 \mathrm{mmol} / \mathrm{L}$ & 70 & 57,1 & 31 & 58,5 & 9 & 52,9 & 0.687 \\
\hline Potássio $<3,5 \mathrm{mmol} / \mathrm{L}$ & 70 & 14,3 & 7 & 13,2 & 3 & 17,6 & 0,696 \\
\hline $\mathrm{CK}>173 \mathrm{U} / \mathrm{L}$ & 42 & 28,6 & 8 & 26,7 & 4 & 33,3 & 0,715 \\
\hline $\mathrm{DHL}>2 \times \mathrm{LSN}^{\ddagger}(\mathrm{U} / \mathrm{L})$ & 62 & 38,7 & 13 & 28,3 & 11 & 68,8 & 0,004 \\
\hline Glicose > $140 \mathrm{mg} / \mathrm{dL}$ & 69 & 27,5 & 13 & 25,0 & 6 & 35,3 & 0.409 \\
\hline $\mathrm{PaO} 2<60 \mathrm{mmHg}$ & 40 & 17.5 & 4 & 13.8 & 3 & 27.3 & 0.369 \\
\hline $\mathrm{PCR}>100 \mathrm{mg} / \mathrm{L}$ & 47 & 74,5 & 27 & 73,0 & 8 & 80,0 & 1,000 \\
\hline
\end{tabular}

$\mathrm{N}$ : Número de casos com dados disponíveis; SNC: Sistema nervoso central; AST: Aspartato aminotransferase; ALT: Alanina aminotransferase; PT: Tempo de protrombina; APTT: Tempo de tromboplastina parcialmente activado; CK: Creatinina quinase; DHL: Lactato desidrogenase sérica; PCR: Proteína C reativa; * Doença renal crónica, neoplasia, diarreia, cianose e hepatomegalia não cumprem requisitos da análise; ${ }^{\dagger}$ Exclui comorbilidades obesidade e dislipidemia; ${ }^{\ddagger}$ nXLSN: Número de vezes acima do limite superior do normal. 
Tabela 2 - Análise uni e multivariada dos achados clínicos e laboratoriais dos doentes internados em unidades de cuidados intermédios/ intensivos ou morte (grupo 2) versus enfermaria geral (grupo 1)

\begin{tabular}{|c|c|c|c|c|c|c|}
\hline \multirow{2}{*}{ Característica* } & \multicolumn{3}{|c|}{ Univariado } & \multicolumn{3}{|c|}{ Multivariado } \\
\hline & OR & IC $95 \%$ & $p$ & OR & IC $95 \%$ & $p$ \\
\hline Tabagismo & 0,14 & $0,02-1,15$ & 0,068 & & & \\
\hline Ausência febre & 16,31 & $1,68-158,40$ & 0,016 & 16,00 & $1,64-156,60$ & 0,017 \\
\hline Exantema petequial & 3,33 & $0,87-12,79$ & 0,079 & & & \\
\hline Vómitos & 0,16 & $0,02-1,34$ & 0,091 & & & \\
\hline Dispneia & 4,36 & $1,18-16,13$ & 0,027 & & & \\
\hline Cefaleias & 0,23 & $0,05-1,10$ & 0,065 & & & \\
\hline In(Creatinina) & 4,76 & $1,44-15,73$ & 0,011 & & & \\
\hline In(Ureia) & 3,55 & $1,35-9,34$ & 0,010 & 3,42 & $1,21-9,68$ & 0,021 \\
\hline DHL & 1,01 & $1,00-1,01$ & 0,011 & & & \\
\hline
\end{tabular}

região contribuído com $30 \%$ dos casos nacionais. ${ }^{1,2}$ Este ano foi considerado um dos anos mais quentes das últimas décadas com valores médios de temperaturas mínimas e máximas superiores à média do período $1971-2000 .^{18}$ Ambientes quentes influenciam positivamente o ciclo reprodutivo do agente e tornam o vetor mais agressivo para o homem pela desidratação a que é sujeito. ${ }^{9}$ Este facto pode ter condicionado a alteração registada no norte do país. O predomínio estival, típico da doença, ${ }^{13,16,19}$ foi constatado nesta série.

A técnica de IF para a deteção de $R$. conorii foi introduzida no HSA-CHP antes do período considerado neste estudo. No entanto, até ao ano 2000 , em todos os casos suspeitos de FEN, a técnica de deteção serológica utilizada foi o Weil-Felix. Pelas suas limitações, ${ }^{13}$ a técnica não foi considerada nesta revisão, pelo que todos os diagnósticos feitos na década de 90 pressupõem o diagnóstico clínico. Por este motivo, o número de casos incluídos na amostra representa apenas uma parcela da totalidade dos casos de infeção assinalados neste hospital.

O facto da Rickettsia conorii se alojar e multiplicar a nível das células endoteliais, justifica o curso desta patologia com o atingimento de diversos sistemas de órgãos. $O$ envolvimento cutâneo da doença, pela presença do exantema maculopapular, foi praticamente universal, o que vai de encontro aos resultados de outros estudos. ${ }^{6,19,20}$ Não foram detetadas apresentações atípicas, nomeadamente escaras de inoculação múltiplas. Apesar da recente descrição de novos agentes em Portugal, como a $R$. conorii ISF, à qual está associada uma menor deteção da escara de inoculação (40\%, aproximadamente), ${ }^{21}$ a frequência deste elemento demonstrou ser semelhante à descrita classicamente. A restante apresentação clínica e laboratorial da doença é paralela com a descrição da literatura. . $19,20,22^{2}$

É interessante constatar que apenas $1 / 4$ dos doentes com avaliação médica prévia foram medicados com a terapêutica apropriada. O facto desta patologia ser pouco frequente e do maior número de casos se registar no interior do país pode refletir-se num baixo contacto dos médicos locais com a doença, explicando o subdiagnóstico; por outro lado, os doentes podem apresentar-se ao médico assistente ainda em fase prodrómica durante a qual, na ausência de contexto epidemiológico, o diagnóstico clínico é praticamente impossível. Curiosamente, a administração pré-hospitalar de doxiciclina não se correlacionou com o desenvolvimento de infeção menos severa. Sabe-se que a instituição terapêutica precoce se associa à prevenção do desenvolvimento de formas graves e antecipação da defervescência dos sintomas, ${ }^{22}$ pelo que este resultado provavelmente está associado ao reduzido número de casos nestas circunstâncias.

A taxa de mortalidade observada coincide com os números descritos noutras séries. ${ }^{11,22} \mathrm{Um}$ dos casos tratava-se de uma idosa com múltiplas comorbilidades e, portanto, com terreno propício ao desenvolvimento de doença severa. Não obstante a associação idade/comorbilidades e mau prognóstico não ter sido demonstrada nesta serie, a literatura sugere que serão factores com impacto. $O$ outro ocorreu num homem de meia-idade com antecedentes de etilismo crónico, sem insuficiência hepática significativa, que evoluiu para disfunção multiorgânica. O efeito imunossupressor do álcool e as frequentes carências nutricionais associadas ao seu consumo sustentado podem condicionar negativamente a resposta imunitária à infeção. De facto, esta foi a única comorbilidade a associar-se a maior tempo de internamento. Apesar de ambos os casos se terem apresentado no serviço de urgência com um tempo de evolução sintomática inferior à média da amostra e, portanto, não ser percetível um atraso na instituição terapêutica, não se pode deixar de ponderar a hipótese do tempo de evolução da doença ter sido mais longo, tendo a sintomatologia surgido apenas mais tardiamente.

Apesar dos crescentes relatos de formas malignas da doença, não foi confirmado o aumento do número de formas 
fatais. Mesmo em 1997, ano em que em Portugal houve um aumento considerável do número de mortes por $\mathrm{FEN},{ }^{4}$ não se sentiram repercussões negativas neste grupo, muito embora seja certo o subdiagnóstico já referido.

Formas severas de FEN, nomeadamente associadas a disfunção neurológica e cardiovascular, ocorreram com frequência. A taxa de severidade da doença foi 1,4 a 4,5 vezes superior à verificada em estudos pregressos, ${ }^{22}$ achado provavelmente associado ao facto deste trabalho avaliar a gravidade da doença exclusivamente em doentes hospitalizados, o que por si só representa uma severidade da doença desproporcional em relação à população avaliada em ambulatório. Por outro lado, não existe um consenso entre estudos no que diz respeito à definição de doença severa, o que se traduz numa limitação na comparação de séries.

São reconhecidos como fatores de mau prognóstico a idade avançada e o atraso na instituição terapêutica. ${ }^{16,22} \mathrm{Nesta}$ série, a idade média dos doentes com doença leve/moderada e com doença severa foi sobreponível. Além disso, o tempo médio de apresentação no serviço de urgência após o início dos sintomas foi semelhante nos dois grupos, pelo que o atraso na procura de cuidados médicos não justifica a maior severidade da doença. Permanece a questão se o primeiro dia de antibioterapia adequada não será mais tardio no grupo com pior prognóstico, apesar do reconhecimento da doença não parecer estar comprometido uma vez que a imagem semiológica da doença é independente da gravidade.

Apirexia à entrada constitui um fator de risco independente para doença mais severa, facto que pode sugerir algum grau de imunocompromisso por parte destes indivíduos. É interessante verificar que esta possível incompetência imunológica não está associada à idade dos doentes, nem às suas comorbilidades, quer em tipo quer em número. À semelhança das descrições de outras séries, a presença de dispneia associou-se mais frequentemente a um internamento em unidades de cuidados diferenciados, ${ }^{22}$ a sugerir um contributo significativo do atingimento directo do endotélio vascular pulmonar ou de disfunção associada ao processo séptico para um curso clínico menos benigno. Paradoxalmente, não se observaram diferenças significativas entre a pressão arterial de oxigénio nos dois grupos.

Doentes que à entrada apresentam lesão renal aguda beneficiam de uma vigilância mais apertada pelo seu pior prognóstico. Uma ureia sérica elevada, consequência da hipoperfusão renal associada à hipotensão do processo séptico, mais do que pelo atingimento direto do rim, ${ }^{23}$ não só se correlaciona com a gravidade da doença como é factor de independente de mau prognóstico. Alterações nos níveis séricos de LDH são praticamente universais, e doseamentos elevados deste marcador demonstram ter valor na identificação de doentes com maior probabilidade de evolução para morte ou internamento em unidades de cuidados hospitalares mais diferenciados.

$O$ facto de não se encontrarem comorbilidades que favoreçam o desenvolvimento de doença complicada e de não se verificarem diferenças significativas em termos da idade e apresentação clínica dos doentes, com a exceção das descritas, levanta questões relativamente aos fatores que contribuem para as formas mais graves da doença. Sabe-se que diferentes subespécies contribuírem de forma díspar para a severidade da doença, facto não esclarecido nesta série uma vez que os métodos serológicos utilizados neste hospital não permitem a identificação de subespécies de $R$. conorii. Por outro lado, o reduzido tamanho da amostra, em particular do grupo com internamento em cuidados intermédios/intensivos ou cujo desfecho foi fatal, bem como a informação incompleta em determinadas variáveis, pode obscurecer fatores humanos contribuidores para doença grave.

Uma limitação deste estudo consiste no facto da avaliação da severidade da doença se basear no serviço de internamento. Apesar de ser indiscutível que os doentes internados em unidades de cuidados intermédios e intensivos tenham um quadro clínico mais severo, face aos internados numa enfermaria geral, é um facto que a gravidade dos casos que requerem cuidados intensivos é diferente da dos que são internados em unidades de cuidados intermédios.

Pela sua natureza retrospetiva e limitações já apontadas, sugere-se que futuros estudos nesta área englobem maior número de casos, tendo como possíveis outcomes a necessidade de internamento em unidades de cuidados intensivos isoladamente, a disfunção de dois ou mais sistemas de órgãos, e/ou a morte, no sentido de clarificar os fatores de mau prognóstico descritos e aferir outros provavelmente encobertos.

\section{CONCLUSÃO}

A FEN é com frequência subdiagnosticada, apesar do diagnóstico ser essencialmente clínico. É necessário um alto grau de suspeição clínica, particularmente em fases precoces da infeção.

A elevada taxa de subnotificação da FEN torna difícil aferir o impacto da patologia no nosso país. Apesar de na maioria dos casos o prognóstico ser favorável, todos os doentes beneficiam de uma vigilância apertada. Independentemente da maioria dos casos ser tratada com sucesso em ambulatório, uma parcela considerável de doentes requer intervenção hospitalar, particularmente os grupos mais fragilizados. Um problema frequente nos serviços de urgência é a atempada identificação dos casos em risco de agravamento clínico. Quase metade dos doentes com FEN desenvolve complicações durante o internamento. A sinalização destes doentes com base na temperatura corporal, presença de dispneia ou lesão renal e níveis séricos de DHL, parâmetros facilmente acessíveis, poderá alterar o curso da doença. Perante a suspeita, a instituição da terapêutica adequada deve ser precoce de modo a evitar internamentos prolongados e evoluções desfavoráveis que acarretam indiscutivelmente custos sociais e económicos.

A sensibilização dos clínicos para esta patologia será provavelmente a pedra de toque para que os doentes com FEN possam ser sinalizados de forma correta e precoce. 


\section{AGRADECIMENTOS}

Os autores agradecem a Paulo Pereira pela disponibilização de informação referente aos métodos serológicos e ao Serviço de Microbiologia do Hospital de Santo António Centro Hospitalar do Porto pela disponibilização de dados.

\section{PROTECÇÃO DE PESSOAS E ANIMAIS}

Os autores declaram que o presente estudo obteve parecer favorável do Conselho de Administração, Gabinete Coordenador de Investigação do Departamento de Ensino, Formação e Investigação/DEFI, Direção Clínica e Comissão de Ética para a Saúde do Centro Hospitalar do Porto.

\section{CONFIDENCIALIDADE DOS DADOS}

Os autores declaram ter seguido os protocolos do seu centro de trabalho acerca da publicação de dados.

\section{CONFLITOS DE INTERESSE}

Os autores declaram a inexistência de conflitos de interesse.

\section{FONTES DE FINANCIAMENTO}

Não existiram fontes de financiamento para a realização deste artigo.

\section{REFERÊNCIAS}

1. Direcção Geral de Saúde. Doenças de declaração obrigatória. 19952008. Publicação da Direcção de Serviços de Epidemiologia e Estatísticas da Saúde/ Divisão epidemiológica. [Consultado 2012 mar 01]. Disponível em: http://www.dgs.pt/.

2. Direcção Geral de Saúde. Doenças de Declaração Obrigatória: Casos Notificados - Portugal 1990- 2010. PORDATA. [Consultado 2012 mar 03]. Disponível em: http://www.pordata.pt/Portugal/Doencas+de+decla racao+obrigatoria+casos+notificados-773

3. Bacellar F, Sousa R, Santos A, Santos-Silva M, Parola P. Boutonneuse fever in Portugal: 1995-2000. Data of a state laboratory. Eur J Epidemiol. 2003;18:275-7.

4. Bacellar F, Beati L, França A, Poças J, Regnery R, Filipe AR. Israeli spotted fever rickettsia (Rickettsia conorii Complex) associated with human disease in Portugal. Emerg Infect Dis. 1999;5:835-6.

5. Walker D, Valbuena G, Olano J. Pathogenic mechanisms of diseases caused by Rickettsia. Ann N Y Acad Sci. 2003;990:1-11.

6. Carmo G, Caixeiro IS, Uva AS, Paiva JD. Febre escaro-nodular. Actualização teórica e análise retrospectiva de 231 casos. Rev Port Doenças Infec. 1981;4:13-28.

7. Brouqui P, Bacellar F, Baranton G, Birtles R, Bjoërsdorff A, Blanco J, et al. Guidelines for the diagnosis of tick-borne bacterial diseases in Europe - ESCMID Study Group Report. Clin Microbiol Infect. 2004;10:1108-32.

8. Bacellar F, Núncio M, Rehacek J, Filipe A. Rickettsiae and Rickettsioses in Portugal. Eur J Epidemiol. 1991;7:291-3.

9. Santos-Silva M, Sousa R, Santos AS, Lopes D, Queijo E, Doreta A, et al Ticks and tick-borne rickettsiae surveillance in Montesinho natural park, Portugal. Ann N Y Acad Sci. 2006;1078:137-42.

10. Rovery C, Brouqui P, Raoult D. Questions on Mediterranean spotted fever a century after its discovery. Emerg Infect Dis. 2008;14:1360-7.

11. Raoult D, Weiller PJ, Chagnon $H$, Chaudet $H$, Gallais $H$, Casanova $P$. Mediterranean spotted fever: clinical, laboratory and epidemiological features of 199 cases. Am J Trop Med Hyg. 1986;35:845-50.

12. Sousa $R$, França $A$, Nóbrega $S$, Belo A, Amaro M, Abreu T, et al. Host - and microbe- related risk factors for and pathophysiology of fatal Rickettsia conorii infection in portuguese patients. J Infect Dis.

2008;198:576-85.

13. Sousa R, Nóbrega S, Bacellar F, Torgal J. Sobre a realidade da febre escaro-nodular em Portugal. Acta Med Port. 2003;16:429-36.

14. Sousa R, Barata C, Vitorino L, Santos-Silva M, Carrapato C, Torgal J, et al. Rickettsia sibirica isolation from a patient and detection in ticks, Portugal. Emerg Infect Dis. 2006;12:1103-8.

15. Brouqui P, Dupont HT, Drancourt M, Bourgeade A, Raoult D. Spotless boutonneuse fever. Clin Infect Dis 1992;14:114-6

16. Sousa R, Nóbrega S, Bacellar F, Torgal J. Mediteranean spotted fever in Portugal: Risk factors for fatal outcome in 105 hospitalized patients. Ann N Y Acad Sci. 2003;990:285-94.

17. Parola P, Paddock D, Raoult D. Tick-borne rickettsioses around the world: emerging diseases challenging old concepts. Clin Microbiol Rev. 2005;18:719-56

18. Instituto Nacional de Meteorologia, IP. Relatório \& Contas 2010 [Consultado 2012 abr 17]. Disponível em: http://www.meteo.pt/export/ sites/default/bin/docs/institucionais/RC2010_IM.pdf

19. Louro E, Campos A, Leitão J, Carvalho A, Santos R, Reis C, et al. Febre escaro-nodular: uma zoonose benigna? Rev Soc Port Med Interna 2006;13:14-8.

20. Raoult D, Parola P. Rickettsia conorii infections (Mediterranean spotted fever, Israeli spotted fever, Indian tick typhus, astrakhan fever). In Rickettsial Diseases. Londres: Informa Healthcare; 2007. p. 125-33.

21. Sousa R, Ismail N, Dória-Nóbrega S, Costa P, Abreu T, França A, et al. The presence of eschars, but not greater severity, in Portuguese patients infected with Israeli spotted fever. Ann N Y Acad Sci. 2005;1063:197202.

22. Botelho-Nevers E, Rovery C, Richet H, Raoult D. Analysis of risk factors for malignant Mediterranean spotted fever indicates that fluoroquinolone treatment has a deleterious effect. J Antimicrob Chemother. 2011:66:1821-30.

23. Raoult D, Parola P. Pathogenesis, immunity, pathology, and pathophysiology in rickettsial diseases. In: Rickettsial Diseases. Londres: Informa Healthcare; 2007. p. 15-24 
Mariana MEIRELES, Rui MAGALHÃES, Arlindo GUIMAS

\section{Febre Escaro-Nodular: Revisão Retrospetiva de Casos Hospitalizados e Fatores Preditores de Doença Severa}

Acta Med Port 2015:28:624-631

Publicado pela Acta Médica Portuguesa, a Revista Científica da Ordem dos Médicos

Av. Almirante Gago Coutinho, 151

1749-084 Lisboa, Portugal.

Tel: +351218428215

E-mail: submissao@actamedicaportuguesa.com

www.actamedicaportuguesa.com

ISSN:0870-399X | e-ISSN: 1646-0758

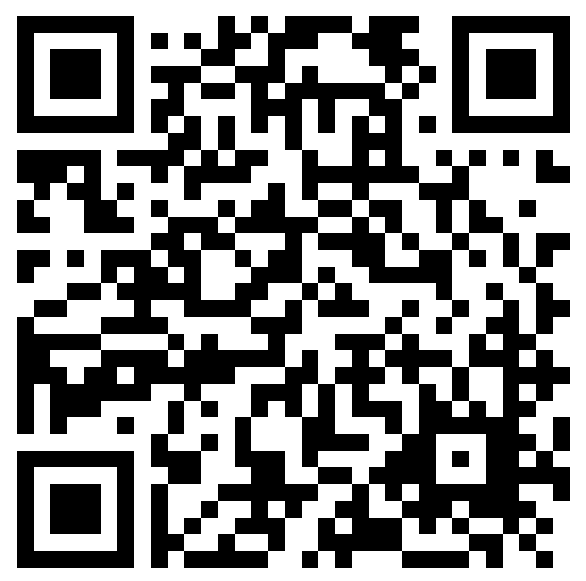

